\title{
The boosting effect of co-transduction with cytokine genes on cancer vaccine therapy using genetically modified dendritic cells expressing tumor-associated antigen
}

\author{
TOSHIYASU OJIMA, MAKOTO IWAHASHI, MASAKI NAKAMURA, KENJI MATSUDA, TEIJI NAKA, \\ MIKIHITO NAKAMORI, KENTARO UEDA, KOICHIRO ISHIDA and HIROKI YAMAUE
}

Second Department of Surgery, Wakayama Medical University, School of Medicine, 811-1 Kimiidera, Wakayama 641-8510, Japan

Received November 15, 2005; Accepted December 29, 2005

\begin{abstract}
The T-helper 1 (Th1) immune reaction is most important in dendritic cell (DC)-based immunotherapy. Interleukin 12 (IL-12) and granulocyte macrophage colonystimulating factor (GM-CSF) play a pivotal role in inducing Th1 and cytotoxic T lymphocyte (CTL) responses. In this study, DCs expressing the natural tumor antigen gp70 of BALB/c-derived CT26 were adenovirally transduced with the IL-12 gene and/or GM-CSF gene, and it was examined whether vaccinations using these genetically engineered DCs can induce strong therapeutic antitumor immunity. Mice were immunized once by subcutaneous (s.c.) injection with genetically modified DCs. The cytotoxic activity of splenocytes against CT26 was assayed in a ${ }^{51} \mathrm{Cr}$-release assay 14 days after immunization. The therapeutic efficacy of the vaccination was examined in s.c. tumor models. The cytotoxic activity of CTLs against CT26 in mice immunized with DCs expressing gp70 (DC-AxCAgp70) was significantly augmented by cotransduction with the GM-CSF/IL-12 gene $(\mathrm{p}<0.0001)$ and remarkably reduced by the depletion of $\mathrm{CD}^{+}$or $\mathrm{CD} 8^{+}$cells $(p<0.01)$. The cytotoxic activity against CT26 of the plain spleen cells in mice immunized with DC-AxCAgp70/GM-CSF/ IL-12 was significantly higher than that in mice immunized with DC-AxCAgp70 ( $<<0.0001)$, and this activity decreased to almost $50 \%$ upon the depletion of NK cells. Vaccinations using DC-AxCAgp70/GM-CSF/IL-12 or DC-AxCAgp70/ IL-12 could elicit potent therapeutic immunity in s.c. tumor models; tumor-free mice were observed in these vaccination groups. However, there was no significant difference between these two groups. A vaccination therapy using DCs cotransduced with the TAA gene and Th 1-type cytokine genes,
\end{abstract}

Correspondence to: Dr M. Iwahashi, Second Department of Surgery, Wakayama Medical University, School of Medicine, 811-1 Kimiidera, Wakayama 641-8510, Japan

E-mail: makoto@wakayama-med.ac.jp

Key words: adenoviral vector, dendritic cells, gp70, granulocyte macrophage colony-stimulating factor, interleukin-12 especially the IL-12 gene, is ideal for immunotherapy in terms of the activation of DCs, NK cells, $\mathrm{CD}^{+}{ }^{+} \mathrm{T}$ cells and $\mathrm{CD}^{+}$ $\mathrm{T}$ cells, and may be useful in the clinical application of a cancer vaccine therapy.

\section{Introduction}

DCs are potent antigen-presenting cells (APCs), capable of inducing a $\mathrm{T}$ cell-dependent immune response (1). Antigenloaded DCs migrate to lymphoid centers where they interact with effector cells and trigger immune responses through the expression of co-stimulatory and adhesion molecules on their surface $(2,3)$.

We have studied a gene-based vaccination strategy using DCs adenovirally transduced with the whole tumor-associated antigen (TAA) gene. This approach has several advantages in that DCs expressing whole TAA produce multiple and/or undefined antigenic epitopes independent of major histocompatibility complex (MHC) alleles, leading to potent antitumor immunity. In fact, we previously reported that DCs transduced with the entire gene encoding endogenous TAA induced the generation of tumor-specific $\mathrm{CD}^{+}$and $\mathrm{CD} 8^{+}$ $\mathrm{T}$ cells, providing an advantage over DCs pulsed with immunodominant peptides in terms of antitumor immune response (4).

The functions of DCs are affected by several immunostimulatory cytokines within the local tissue environment (5). In particular, GM-CSF is a potent stimulator of DCs (6). We also previously reported that a vaccination using DCs cotransduced with TAA and GM-CSF enhanced in vivo functions of DCs, especially the migratory capacity for draining the lymph node by up-regulation of $\mathrm{CC}$ chemokine receptor 7 (CCR7) expression, and achieved a more efficient tumor suppression in mouse models than a vaccination using DCs expressing TAA only (7). However, these findings were not reflected in the survival advantage, and no mice were free of tumors although the in vivo treatment model was a single subcutaneous immunization with genetically modified DCs. Considering the potential clinical applications of this strategy, additional devices are necessary to achieve a satisfactory response.

In DC-based immunotherapy, the Th1 immune reaction is most important to activate CTLs. IL-12 is a key cytokine in 
immune regulation, originally identified as natural killer cell stimulatory factor, and is important for the induction of Th1 and CTL immunity (8-10). IL-12 presumably acts on Th1 induction as a potent enhancer of interferon (IFN)- $\gamma$ production by DCs $(11,12)$. In addition, IL-12 and IFN- $\gamma$ derived from DCs probably influence innate immunity such as responses of NK and NKT cells. DCs play a predominant role in the activation of NK cells, and NK cells appearing early in tumor lesions play a pivotal role in the innate immunity against tumor cells (13-15). When mice bearing liver tumors were immunized with an adenoviral vector expressing IL-12 intratumorally, a regression of metastatic lesions in the lung occurred through the activation of both $\mathrm{T}$ cells and NK cells (14). Therefore, we decided that a vaccination using genetically modified DCs co-transduced with the TAA gene and IL-12 gene would elicit an ideal antitumor immune response by activating both NK cells and tumor-specific CTLs.

In this study, murine DCs expressing the natural tumor antigen gp70 of BALB/c-derived CT26 were adenovirally transduced with both the IL-12 gene and GM-CSF gene, and it was examined whether vaccinations using these genetically engineered DCs induce a much stronger therapeutic antitumor immunity against CT26 tumors than vaccinations using DCs expressing gp70 alone (16). Furthermore, we investigated the mechanism of the boosting effects of co-transduction of DCs with the IL-12 and GM-CSF genes.

\section{Materials and methods}

Mice and cell lines. Female 6- to 8-week old BALB/c mice $\left(\mathrm{H}-2^{\mathrm{d}}\right)$ (Japan SLC Inc., Hamamatsu) were used for the experiments. All animal experiments were performed in accordance with the Japanese Government's Animal Protection and Management Law (no. 105) and Notification on Feeding and Safekeeping of Animals (no. 6), and the guidelines for animal experiments of Wakayama Medical University. The syngeneic tumor cell line, CT26 $\left(\mathrm{H}-2^{\mathrm{d}}\right)$, an undifferentiated low-immunogenic murine colorectal adenocarcinoma cell line (kindly provided by Hoffman-La Roche, Kamakura, Japan) and human embryonic kidney cell line, 293 (American Type Culture Collection, Rockville, MD, USA), were grown in DMEM (Nissui Pharmaceutical Co, Tokyo, Japan) supplemented with $10 \%$ fetal bovine serum (FBS) (Invitrogen, Carlsbad, CA, USA), 2 mM L-glutamine (Invitrogen), $100 \mathrm{U} / \mathrm{ml}$ penicillin and $100 \mu \mathrm{g} / \mathrm{ml}$ streptomycin (Invitrogen). Meth-A $\left(\mathrm{H}-2^{\mathrm{d}}\right)$, a methylcholanthrene-induced sarcoma cell line (RIKEN BioResource Center, Ibaraki, Japan), and SP2/0 (H-2 $\left.{ }^{\mathrm{d}}\right)$, a myeloma cell line (RIKEN), were grown in RPMI-1640 medium (Nissui) supplemented with 10\% FBS, $2 \mathrm{mM}$ L-glutamine, $100 \mathrm{U} / \mathrm{ml}$ penicillin and $100 \mu \mathrm{g} / \mathrm{ml}$ streptomycin.

Recombinant adenoviral vectors. Recombinant AxCAgp70, AxCAGM-CSF and AxCALacZ were generated by the COSTPC method as previously described $(7,17,18)$. AxCAp35 (Adex1 CAmIL12p35) and AxCAp40 (Adex1 CAmIL12p40), which express murine IL-12, were obtained from RIKEN BioResource Center. The titer of virus was measured by standard plaque assay in 293 cells (19).
Generation of immature DCs from bone marrow. For the generation of DCs and induction of CTLs, RPMI-1640 medium supplemented with $10 \%$ FBS, 2 mM L-glutamine, $50 \mu \mathrm{M}$ 2-mercaptoethanol (Invitrogen), $100 \mathrm{U} / \mathrm{ml}$ penicillin and $100 \mu \mathrm{g} / \mathrm{ml}$ streptomycin was used (complete medium). DCs were prepared from murine bone marrow precursors as described previously $(7,20)$. Briefly, murine bone marrow cells were cultured in complete medium containing $200 \mathrm{U} / \mathrm{ml}$ recombinant murine (rm) GM-CSF (Kirin Brewery Co., Tokyo, Japan) for 10 days. On day 10, nonadherent cells were collected and used as immature DCs (iDCs). These DC preparations were stained with FITC-conjugated monoclonal antibodies against murine cell surface molecules, and examined by flow cytometry. Approximately $90 \%$ of the cells showed high levels of CD11c (HL3), more than 99\% of the cells showed high levels of $\mathrm{H}-2^{\mathrm{d}}$ (SF1-1.1) and I-A $\mathrm{A}^{\mathrm{d}}$ (AMS-32.1), and approximately $10 \%$ of the cells showed low levels of CD40 (3/23) and CD86 (GL1) (all from BD PharMingen, San Diego, CA, USA) (data not shown).

Adenoviral vector-mediated gene transfer into DCs. Immature DCs were transfected with each recombinant adenoviral (Ad) vector using a centrifugal method as described previously (7). Briefly, DCs were mixed with Ad vectors at a multiplicity of infection (MOI), and centrifuged at $2000 \mathrm{xg}$ at $37^{\circ} \mathrm{C}$ for $2 \mathrm{~h}$. Our previous study showed that the optimal MOI for AxCAgp70 and AxCAGM-CSF was 100 and 5, respectively, in terms of the induction of CTL activity (7). Therefore, the MOI values of these vectors were fixed in this study. To determine the optimal dose of Ad vectors expressing IL-12, DCs were transfected simultaneously with AxCAgp70 (MOI of 100) and AxCAp35/AxCAp40 (MOI of 1/MOI of 1.5, 2/3, $40 / 60)$. DCs were then placed at $2 \times 10^{6} /$ well in $10 \mathrm{ml}$ of complete medium with $10 \mu \mathrm{g} / \mathrm{ml}$ of lipopolysaccharide (LPS) (Escherichia coli 0127:B8; Sigma, St. Louis, MO, USA). After 48 h, DCs were used for the experiments. Abbreviations for the vectors used are: DC-AxCAgp70, DCs transfected with AxCAgp70; and DC-AxCAgp70/GM-CSF/IL-12, DCs transfected with AxCAgp70, AxCAGM-CSF and AxCAp35/AxCAp40. Approximately 10\% of immature DCs showed low levels of CD40 and CD86. Mature DCs induced by treatment with LPS showed augmented expression of CD40 (75\%) and CD86 (30\%). The expression of co-stimulatory molecules in DCs was induced by adenoviral transduction, and markedly augmented by co-transduction with the IL-12 gene; DC-AxCAgp70/IL-12 showed 84\% CD40 and 56\% CD86 (data not shown).

Generation of CTLs by in vitro restimulation with target cells and cytotoxicity assay. To determine whether the antitumor activity induced by immunization with DCs expressing gp70 would be enhanced by co-transduction of the GM-CSF and IL-12 genes, DCs were simultaneously transfected with AxCAgp70 and AxCAGM-CSF and AxCAp35/AxCAp40. $\mathrm{BALB} / \mathrm{c}$ mice were immunized once by s.c. injection of $5 \times 10^{5}$ genetically modified DCs suspended in $200 \mu 1$ of PBS. Spleen cells were isolated 14 days after the DC immunization, and the in vivo-primed splenocytes were pooled and cocultured $\left(4 \times 10^{6} / \mathrm{ml}\right)$ with irradiated $(10,000 \mathrm{rads})$ CT26 cells $\left(4 \times 10^{5} / \mathrm{ml}\right)$ in a 6 -well plate $(4 \mathrm{ml} /$ well $)$ with $\mathrm{rmIL}-2$ 
$50 \mathrm{IU} / \mathrm{ml}$ (BD PharMingen). To investigate the cytotoxic activity of the unstimulated spleen cells in mice immunized with genetically modified DCs, we also used plain splenocytes without in vitro restimulation as effector cells. After 5 days of co-culture, the in vitro-restimulated splenocytes or spleen cells (harvested in complete medium with rmIL-2 $50 \mathrm{IU} / \mathrm{ml}$ without CT26 for 3 days) were assayed using a $4-\mathrm{h}{ }^{51} \mathrm{Cr}-$ release assay as described previously $(7,21)$.

Antibody blocking assay. To assess the functional phenotypes of CTLs generated by the immunization of genetically engineered DCs, the effector cells or target cells were incubated with anti-mouse CD4 (H129.19; BD PharMingen), antimouse CD8a (53-6.7; BD PharMingen), anti-mouse $\mathrm{H}-2^{\mathrm{d}}$ (SF1-1.1; BD PharMingen), or rat immunoglobulin G (IgG) (control antibody; Sigma) at a dose of $10 \mu \mathrm{g} /$ well for $30 \mathrm{~min}$ at $4^{\circ} \mathrm{C}$ before a $4-\mathrm{h}^{51} \mathrm{Cr}$-release assay.

In vivo NK cell or T cell subset depletion assay. To investigate the mechanism of the cytotoxic activity against CT26 induced by the immunization of genetically engineered DCs, $\mathrm{NK}$ cells or $\mathrm{CD}^{+} / \mathrm{CD}^{+} \mathrm{T}$ cells were depleted in vivo before immunization. The mice were intraperitoneally injected with rabbit anti-asialo GM1 (Wako Pure Chemical Industries, Osaka, Japan) (20 $\mu \mathrm{g}$ per mouse on days $-3,0,+3,+6,+9$, and +12$)$, PBS, or control rabbit serum (Sigma) (200 $\mu 1$ per mouse on days $-3,0,+3,+6,+9$, and +12$)$, anti-CD4 (H129.19; BD PharMingen), anti-CD8a (53-6.7; BD PharMingen), or control rat IgG (Sigma) (200 $\mu \mathrm{g}$ per mouse on days $-3,0,+3,+6$, and +9 ) (8). Our flow cytometric analyses showed that $>99 \%$ of the targeted cells were specifically depleted (data not shown). On day 0, immunodepleted mice ( $n=4 /$ group) were immunized once with DC-AxCAgp70/GM-CSF/IL-12. Fourteen days after the immunization of asialo GM1-treated mice, spleen cells were harvested and cultured in complete medium with rmIL-2 $50 \mathrm{IU} / \mathrm{ml}$ without CT26 for 3 days, and cytotoxic activity against CT26 and SP2/0 was tested in a $4-\mathrm{h}{ }^{51} \mathrm{Cr}$-release assay. On the other hand, 14 days after the immunization of anti-CD4- or CD8-treated mice, splenocytes were restimulated with CT26 in vitro for 5 days, and CTL activity against CT26 was tested in a $4-\mathrm{h}{ }^{51} \mathrm{Cr}-$ release assay.

Assay for cytokine secretion. Genetically modified DCs were seeded at a concentration of $5 \times 10^{5}$ cells/well and cultured for 48 h. Supernatants were then harvested, and murine IFN- $\gamma$ and IL-12 (p70) levels were measured using mIFN- $\gamma$ ELISA and mIL-12 p70 ELISA kits (Endogen, Woburn, MA, USA).

Experiment design of in vivo antitumor therapy for s.c. tumor models. BALB/c mice were inoculated subcutaneously in the right flank with $1 \times 10^{6} \mathrm{CT} 26$. After 5 days, tumor-bearing mice ( $n=6 /$ group) were injected s.c. in the opposite flank with $5 \times 10^{5}$ genetically modified DCs: group 1, PBS; group 2, DC-AxCALacZ; group 3, DC-AxCAgp70; group 4, DCAxCAgp70/IL-12; group 5, DC-AxCAgp70/GM-CSF; and group 6, DC-AxCAgp70/GM-CSF/IL-12. When the tumor diameter exceeded $15 \mathrm{~mm}$, tumor-bearing mice were sacrificed. Otherwise, 19 days after treatment, all mice were sacrificed and the size of the s.c. tumor was estimated using the following formula: (short diameter) ${ }^{2} \mathrm{x}$ long diameter $\mathrm{x} 0.52$.

Statistical analysis. StatView 5.0 software (Abacus Concepts, Inc., Berkeley, CA, USA) was used for all statistical analyses. Quantitative results are expressed as the mean \pm SD. Statistical analysis was performed with ANOVA and Fisher's test. A p-value of $<0.05$ was considered to be significant.

\section{Results}

CTL responses induced by immunization with DCs expressing the TAA gene, GM-CSF gene and/or IL-12 gene. We first examined the optimal MOI of Ad vectors AxCAp35 and AxCAp40 expressing IL-12 in terms of enhancing CTL activity. The CTL activity of spleen cells against CT26 in mice immunized with DCs expressing murine TAA gp70 was significantly enhanced by co-transduction of the IL-12 gene, and the optimal dose of AxCAp35 and AxCAp40 was suggested to be an MOI of 2 and 3, respectively (data not shown). In this study, the dose of AxCAIL-12 was fixed at an MOI of 2 for AxCAp35 and an MOI of 3 for AxCAp40. The CTL activity of spleen cells against CT26 induced by immunization with DCs expressing gp70 was compared to those with DCs simultaneously expressing the gp70 gene, GM-CSF gene and/or IL-12 gene. The cytotoxic activity against CT26 in mice immunized with DC-AxCAgp70 was significantly augmented by co-transduction with the GM-CSF gene and/or IL-12 gene, especially in the mice immunized with DCs simultaneously expressing gp70, GM-CSF and IL-12 ( $<<0.0001$; Fig. 1A). On the other hand, the CTL activity against Meth-A, which does not express gp70, was $<5 \%$ in all groups (data not shown). The CTL activity of spleen cells against CT26 induced by immunization with genetically engineered DCs simultaneously expressing gp70, GM-CSF and IL-12 was completely blocked by anti-CD 8 antibody and anti-H-2 ${ }^{\mathrm{d}}$ antibody, suggesting that these genetically engineered DCs induced an MHC class I-restricted TAA-specific $\mathrm{CD}^{+}$ CTL response ( $<<0.0001$; Fig. 1B).

In vivo $T$ cell subset depletion assay. We examined the role of $\mathrm{CD}^{+}$and $\mathrm{CD}^{+} \mathrm{T}$ cells in the induction of CTL responses against CT26 in mice immunized with DCs expressing gp70 and GM-CSF/IL-12 by depleting each cell in vivo with anti-CD4 or anti-CD8 monoclonal antibodies. As shown in Fig. 1C, immunodepletion of $\mathrm{CD}^{+}$or $\mathrm{CD}^{+}$cells resulted in a significant reduction in cytotoxic activity of CTLs against CT26 compared to controls (anti-CD4, p $<0.0001$; anti-CD $8, \mathrm{p}<0.01)$. Of note, the impact of $\mathrm{CD}^{+}$cell depletion was stronger than that of $\mathrm{CD}^{+}$cell depletion in terms of the reduction in cytotoxicity (anti-CD4 vs. antiCD8, $\mathrm{p}<0.01)$.

Cytotoxic activity against CT26 of spleen cells without in vitro restimulation by CT26. The cytotoxic activity against CT26 of the spleen cells not restimulated with CT26 in vitro in mice immunized with DC-AxCAgp70 or DC-AxCAgp70/ GM-CSF was as low as that in mice immunized with DCAxCALacZ. However, the activity in mice immunized with 

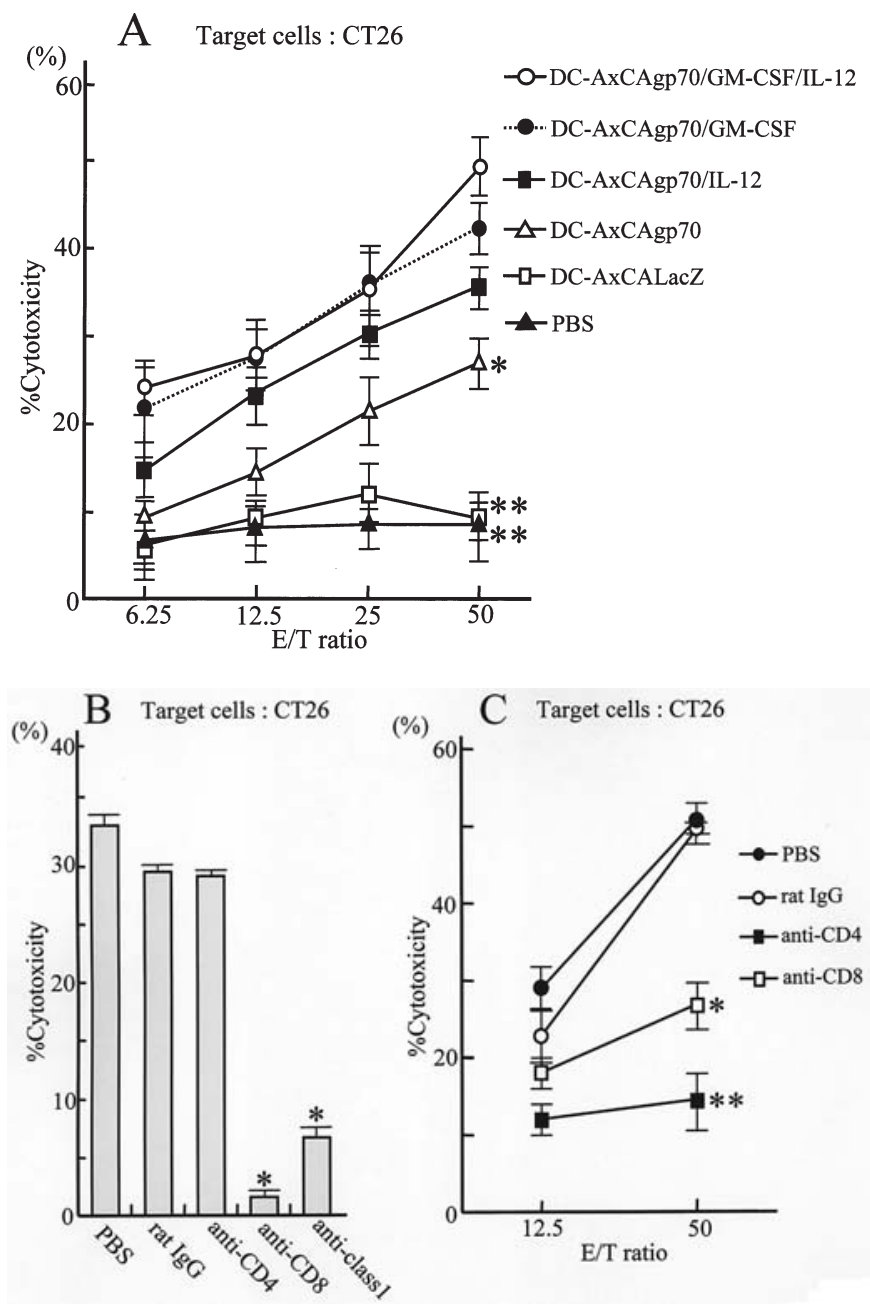

Figure 1. (A) CTL activity induced by immunization using genetically modified DCs. BALB/c mice were immunized once by s.c. injection of DCs with: DC-AxCAgp70 ( $\triangle$ ), DC-AxCAgp70/IL-12 (•), DC-AxCAgp70/GM-


Fourteen days after immunization, spleen cells were restimulated in vitro for 5 days with CT26. CT26 was used as a target. Results are shown as the mean $\pm \mathrm{SD}(\mathrm{n}=5$ for each group). *Significantly different from DCAxCAgp70/GM-CSF/IL-12 and DC-AxCAgp70/GM-CSF (E/T: 50, $\mathrm{p}<0.0001)$ and from DC-AxCAgp70/IL-12 $(\mathrm{p}<0.001) .{ }^{* *}$ Significantly different from DC-AxCAgp70 ( $<<0.0001)$. (B) Antibody blocking assay. Blocking the CTL activity induced by DC-AxCAgp70/GM-CSF/IL-12 was achieved by incubating effector cells or target cells with anti-CD4 (antiCD4), anti-CD8a (anti-CD8), anti-H-2 ${ }^{\mathrm{d}}$ (anti-class I), and control rat $\mathrm{IgG}$ (rat $\mathrm{IgG}$ ) antibodies before a $4-\mathrm{h}{ }^{51} \mathrm{Cr}$-release assay. CT26 was used as a target. Results are shown as the mean $\pm \mathrm{SD}(\mathrm{n}=3$ for each group, $\mathrm{E} / \mathrm{T}$ : 25$)$. ${ }^{*}$ Significantly different from PBS and rat $\operatorname{IgG}(\mathrm{p}<0.0001)$. (C) In vivo $\mathrm{T}$ cell depletion assay. Mice were treated with anti-CD4, anti-CD8 or rat IgG, and then immunized once with DC-AxCAgp70/GM-CSF/IL-12. Fourteen days after immunization, splenocytes were restimulated in vitro with CT26, and CTL activity was tested in a 4-h ${ }^{51} \mathrm{Cr}$-release assay. CT26 was used as a target. Results are shown as the mean $\pm \mathrm{SD}$ ( $\mathrm{n}=4$ for each group). ${ }^{* *}$ Significantly different from PBS and rat $\operatorname{IgG}(\mathrm{p}<0.0001)$ and ${ }^{*}$ significantly different from PBS and rat IgG $(\mathrm{p}<0.01)$. The difference between anti-CD4 and anti-CD8 was significant $(\mathrm{p}<0.01)$.

DC-AxCAgp70/GM-CSF/IL-12 was remarkably greater than that in mice immunized with DC-AxCAgp70 or DCAxCAgp70/GM-CSF ( $<<0.0001$; Fig. 2A). The cytotoxic activity against $\mathrm{SP} 2 / 0$ of the spleen cells in mice immunized with DC-AxCAgp70/GM-CSF/IL-12 was significantly higher than that in mice immunized with DC-AxCAgp70 or
A Target cells : CT26 B Target cells : SP2/0 C Target cells : Meth-A
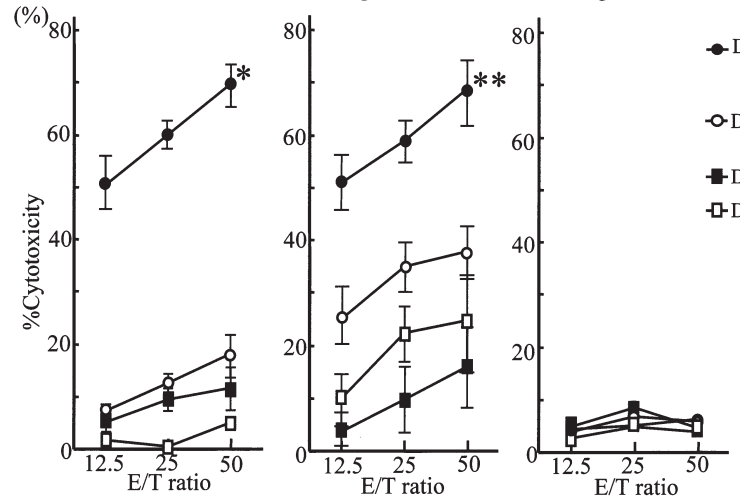

Figure 2. Cytotoxic activity of the spleen cells in mice immunized with genetically modified DCs expressing TAA, GM-CSF and IL-12. BALB/c mice were immunized once by s.c. injection of DC-AxCAgp70/GMCSF/IL-12 (•), DC-AxCAgp70/GM-CSF (॰), DC-AxCAgp70 (•), and DCAxCALacZ ( $\square$ ). Fourteen days after immunization, spleen cells were harvested in complete medium, and incubated without CT26 for 3 days. The cytotoxic activity was then examined by ${ }^{51} \mathrm{Cr}$-release assay. CT26 (A), SP2/0 (B) and Meth-A (C) were used as target cells. Results are shown as the mean $\pm \mathrm{SD}$ ( $\mathrm{n}=5$ for each group). (A) *Significantly different from DCAxCAgp70, DC-AxCAgp70/GM-CSF and DC-AxCALacZ ( $<<0.0001$ ). (B) ${ }^{* *}$ Significantly different from DC-AxCAgp70, DC-AxCAgp70/GM-CSF and DC-AxCALacZ $(\mathrm{p}<0.001)$.
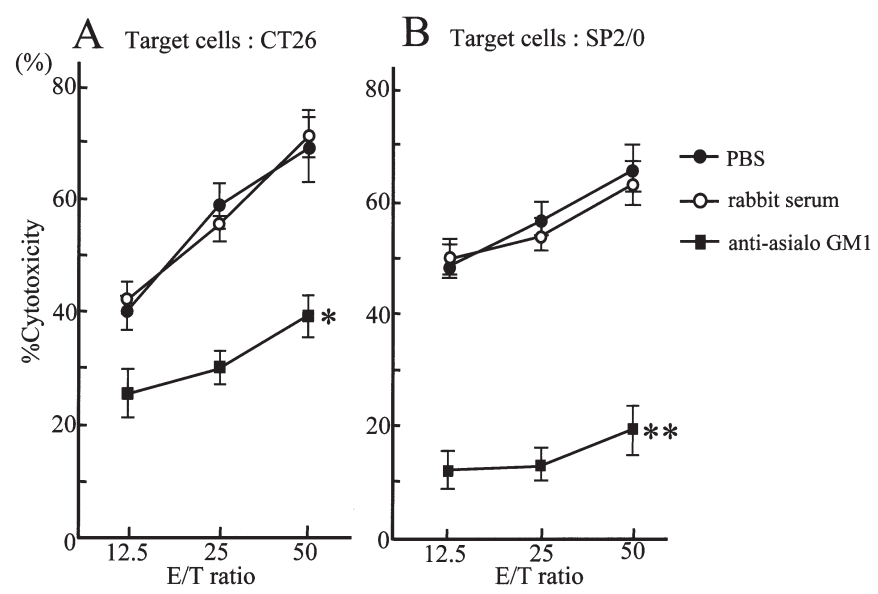

Figure 3. In vivo NK cell depletion assay. Mice were treated with various antibodies and then immunized once with DC-AxCAgp70/GM-CSF/IL-12. Fourteen days after immunization, spleen cells were harvested, cultured for 3 days without CT26, and cytotoxic activity was examined by ${ }^{51} \mathrm{Cr}$-release assay. CT26 (A) and SP2/0 (B) were used as target cells. Results are shown as the mean \pm SD ( $n=4$ for each group). (A) *Significantly different from PBS and rabbit serum $(\mathrm{p}<0.01)$. (B) ${ }^{*}$ Significantly different from PBS and rabbit serum $(\mathrm{p}<0.001)$.

DC-AxCAgp70/GM-CSF ( $<<0.001$; Fig. 2B). On the other hand, the cytotoxic activity against Meth-A was low in all groups (Fig. 2C). Accordingly, it was suggested that the NK activity in mice immunized with DCs simultaneously expressing gp70 and GM-CSF was strongly enhanced by cotransduction of the IL-12 gene.

In vivo NK depletion assay. To determine the role of the NK cells in the cytotoxic activity against CT26 of the spleen cells in mice immunized with DCs expressing gp70 and GM-CSF/ 


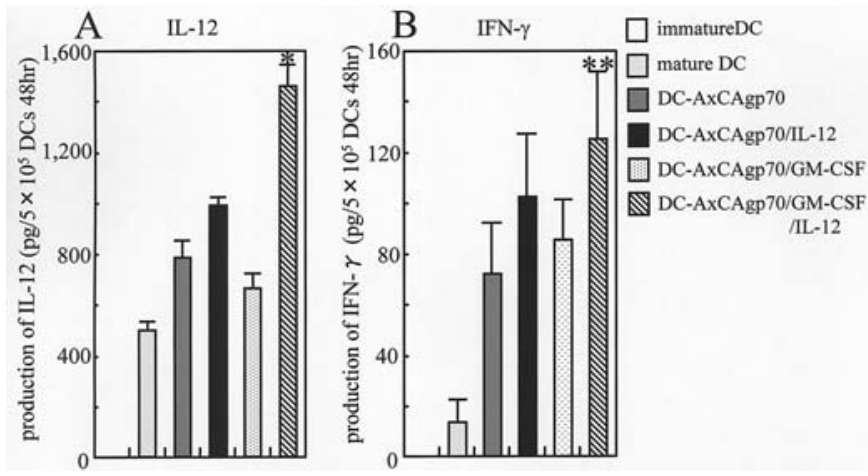

Figure 4. Secretion of Th 1-type cytokines from genetically modified DCs. Immature DCs, LPS-stimulated DCs (mature DC), or genetically modified DCs were plated on a 48 -well plate $\left(5 \times 10^{5}\right.$ cells/well), and each well was filled to a final volume of $1 \mathrm{ml}$ with complete medium. After incubation at $37^{\circ} \mathrm{C}$ for $48 \mathrm{~h}$, supernatants were collected and measured with ELISA kits specific for respective cytokines (A, IL-12; B, IFN- $\gamma$ ). Results are shown as the mean $\pm \mathrm{SD}$ ( $\mathrm{n}=3$ for each group). (A) *Significantly different from mature DC, DC-AxCAgp70, DC-AxCAgp70/IL-12 and DC-AxCAgp70/ GM-CSF $(p<0.0001)$. (B) ${ }^{* *}$ Significantly different from mature $\mathrm{DC}(\mathrm{p}<0.001)$, and from DC-AxCAgp70, DC-AxCAgp70/GM-CSF ( $\mathrm{p}<0.05)$.

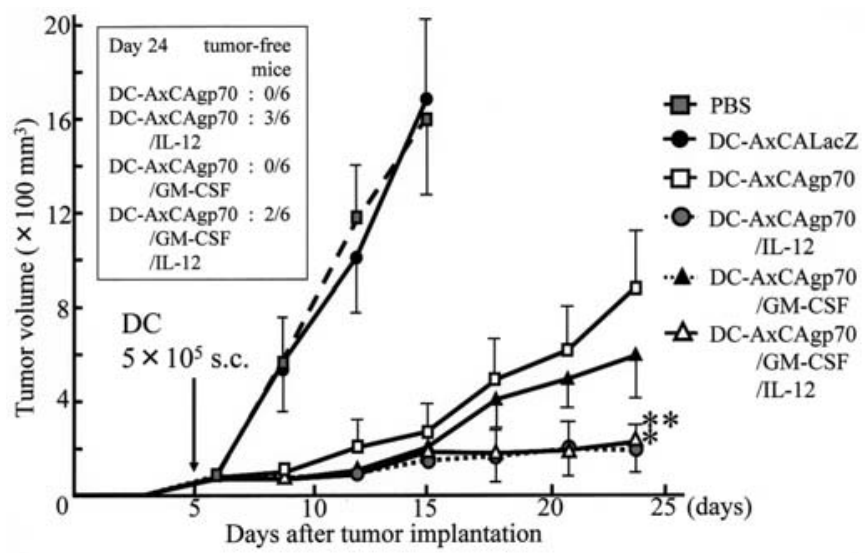

Figure 5. Tumor growth suppression in mice immunized with genetically modified DCs in s.c. tumor models. BALB/c mice were inoculated subcutaneously in the right flank with $1 \times 10^{6} \mathrm{CT} 26$ cells. Five days later, tumor-bearing mice ( $n=6$ for each group) were treated with s.c. injection in the opposite flank of $5 \times 10^{5}$ DC-AxCALacZ (•), DC-AxCAgp70 ( $\square$ ), DCAxCAgp70/IL-12 (grey circle), DC-AxCAgp70/GM-CSF (^), DCAxCAgp70/GM-CSF/IL-12 $(\triangle)$, or PBS (grey square). Results are presented as mean tumor volumes in each group. ${ }^{* *}$ Significantly different from PBS, DC-AxCALacZ (day 15, p<0.0001). *Significantly different from DCAxCAgp70 (day 24, p<0.01), and from DC-AxCAgp70/GM-CSF (day 24, $\mathrm{p}<0.05)$. The difference between DC-AxCAgp70/GM-CSF/IL-12 and DCAxCAgp70/IL-12 was not significant.

IL-12, mice were injected with anti-asialo GM1 antibody to deplete NK cells in vivo. The cytotoxic activity against CT26 of the spleen cells in anti-asialo GM1-treated mice decreased to almost half of that in mice treated with control rabbit serum or PBS ( $<<0.01$; Fig. $3 \mathrm{~A})$. In contrast, the cytotoxic activity against SP2/0 of the spleen cells in anti-asialo GM1-treated mice was almost eliminated ( $<<0.001 ;$ Fig. 3B).

Secretion of Th 1-type cytokines from genetically modified DCs. Although neither IL-12 nor IFN- $\gamma$ was detected in immature DCs, mature DCs produced some IL-12 and IFN- $\gamma$. Adenoviral transduction of the gp70 gene increased the amount of these cytokines, and co-transduction of the IL-12 gene enhanced the production further. Interestingly, DCs transduced simultaneously with both the GM-CSF gene and IL-12 gene produced significantly augmented levels of not only IL-12, but also IFN- $\gamma(p<0.0001$ and $p<0.05$; Fig. 4 A and B $)$.

Therapeutic efficacy of genetically modified DCs in s.c. tumor models. We examined the therapeutic efficacy of vaccinations using genetically modified DCs simultaneously expressing gp70, GM-CSF and/or IL-12 in vivo. A single vaccination using DC-AxCAgp70, DC-AxCAgp70/IL-12, DC-AxCAgp70/GM-CSF or DC-AxCAgp70/GM-CSF/IL-12 showed remarkable therapeutic efficacy in this pre-existing tumor model $(\mathrm{p}<0.0001)$. In particular, the vaccination of DC-AxCAgp70/IL-12 or DC-AxCAgp70/GM-CSF/IL-12 elicited a more potent efficacy than that of DC-AxCAgp70 or DC-AxCAgp70/GM-CSF ( $<<0.05)$, and tumor-free mice were observed in the DC-AxCAgp70/GM-CSF/IL-12 (2/6) and DC-AxCAgp70/IL-12 (3/6) vaccination groups on day 24 (Fig. 5). However, there was no significant difference between these two vaccination groups (Fig. 5).

\section{Discussion}

The activation of Th1-type immunity is crucial for tumor immunotherapy in order to overcome the immunosuppressive conditions in a tumor-bearing host, as a Th1-biased cytokine balance regulated by IL-12 and IFN- $\gamma$ is desirable for not only sensitizing CTLs to TAA via APCs, but also activating innate immunity involving NK cells and NKT cells $(14,22)$. Therefore, in this study, genetically modified murine DCs expressing the natural tumor antigen gp70 and GM-CSF were adenovirally co-transduced with the IL-12 gene to in vivo augment the antitumor immunity induced by vaccinations using these DCs. Our results showed that the vaccination using DCs simultaneously expressing gp70, GM-CSF and IL-12 elicited a potent therapeutic immunity in s.c. tumor models, and tumor-free mice were observed in the group vaccinated with these DCs. Moreover, the antitumor immune responses in those mice involved both an NK cell-mediated innate response and a tumor-specific acquired $\mathrm{T}$ cell response.

Analyses of immunological characteristics of DCs are imperative for the development of DC-based immunotherapy because the polarity of the immune response is greatly influenced by the activity and maturity of DCs during T cell sensitization $(23,24)$. It is well known that GM-CSF is indispensable for the differentiation and activation of DCs $(25,26)$, and our previous study has also shown that GM-CSF enhances the $\mathrm{T}$ cell response through the activation of $\mathrm{DC}$ functions, especially the capacity to migrate to draining lymph nodes (7). It has been demonstrated that IL-12 genetransduced human DCs display a more mature phenotype than non-transduced DCs, as judged by increased expression of CD83, CD80, CD86, and MHC class I and II molecules, and those DCs were significantly superior to non-transduced DCs in terms of the stimulation of autologous and allogenic T lymphocyte responses (27). In this study, the expression of co-stimulatory molecules on DCs adenovirally transduced with the IL-12 gene was markedly enhanced, and DCs 
transduced simultaneously with the GM-CSF gene and IL-12 gene produced high levels of IFN- $\gamma$ and IL-12, suggesting the presence of an autocrine-positive feedback pathway of transduced cytokines around DCs.

NK cells are efficient innate killers of tumor cells, especially during the early phase of tumorigenesis. NK cells also directly participate in acquired immune responses, primarily by interacting with DCs (28). It has been reported that human DCs, induced to mature by LPS, increased the expression of CD69 on peripheral blood NK cells and enhanced the cytotoxic activity of NK cells against NKsensitive target cells and NK-resistant target cells (29). The importance of the DC-NK cell interaction in tumor rejection is suggested by the finding that injected DCs augment NK cell-mediated antitumor immunity in tumor-bearing mice (30). The interaction between DC and NK cells is also established in vitro (30). In the present study, the cytotoxic activity against CT26 of spleen cells in mice vaccinated with DCs expressing gp70 and GM-CSF/IL-12 was remarkably reduced by the depletion of NK cells. This suggests that genetically modified DCs can activate NK cells through IL-12 and GMCSF produced by DCs in the microenvironment, and that activated NK cells could play an important role as efficient killers of tumor cells in vivo.

Our results suggest that Th 1-type cytokines could augment the sensitivity of $\mathrm{CD}^{+}$CTLs specific for TAA. CD8 ${ }^{+}$and $\mathrm{CD}^{+} \mathrm{T}$ lymphocytes are often essential for humoral and cellular immune responses, and $\mathrm{CD}^{+} \mathrm{T}$ helper $\left(\mathrm{CD} 4^{+} \mathrm{Th}\right)$ cell responses play a particularly vital role in immunologically mediated tumor regression (31). $\mathrm{CD}^{+}{ }^{+} \mathrm{Th}$ cells can recognize helper peptides presented in the context of MHC class II molecules on the surface of DCs, and CD4 ${ }^{+}$Th1 cells can support the priming of $\mathrm{CD}^{+}$tumor-reactive $\mathrm{T}$ cells $(32,33)$. Our previous study demonstrated that vaccination using DCs transduced with the whole TAA gene can generate not only TAA-specific $\mathrm{CD}^{+} \mathrm{T}$ cells, but also $\mathrm{CD} 4^{+} \mathrm{T}$ cells (4). Moreover, it has been reported that when mice bearing s.c. tumors were intratumorally immunized with DCs adenovirally transduced with IL-12 and IL-18 genes, $\mathrm{CD} 4^{+}$and $\mathrm{CD} 8^{+} \mathrm{T}$ cells were required for tumor rejection (34). IL-12 polarizes CD4 ${ }^{+}$ Th cell responses toward Th1-type immunity $(34,35)$. In concordance with previous reports, we considered that DCs transduced with the gp70 and GM-CSF/IL-12 genes can endogenously express gp70-specific helper peptides restricted MHC class II, and the Th 1-biasing cytokine IL-12 produced by the DCs can enhance antigen presentation from these DCs to $\mathrm{CD}^{+}{ }^{+} \mathrm{Th} 1$ cells. In this study, it was demonstrated that the generation of gp70-specific CD8 ${ }^{+}$CTLs was dependent on $\mathrm{CD}^{+} \mathrm{Th}$ cells, and the impact of $\mathrm{CD}^{+} \mathrm{T}$ cell depletion was stronger than that of $\mathrm{CD}^{+} \mathrm{T}$ cell depletion in terms of reduction of cytotoxicity against CT26.

The cytotoxic activity against CT26 induced by the immunization of DCs co-transduced with the gp70 and GM-CSF/IL-12 genes was significantly higher than that of mice immunized with DC-AxCAgp70/IL-12. Nevertheless, in s.c. tumor models, there was no difference in therapeutic efficacy between the DC-AxCAgp70/GM-CSF/IL-12 and DC-AxCAgp70/IL-12 vaccination groups, contrary to our expectations. Several reasons can be provided. First, as we previously described, GM-CSF gene co-transduction of DCs enhanced the migratory capacity of DCs with up-regulation of CCR7 expression (7). Our preliminary data showed that the CCR7 expression in DCs was augmented by adenoviral co-transduction, not only with the GM-CSF gene but also with the IL-12 gene, suggesting that IL-12 also plays a crucial role in the migratory capacity of DCs to draining lymph nodes in which DCs can stimulate T cells, leading to an enhanced in vivo tumor growth suppression (data not shown). Second, it is generally accepted that CT26 cells have a moderate sensitivity to NK cells (36). It is considered that NK activity is strongly enhanced by IL-12, explaining why there was no difference in antitumor effects against CT26 between mice immunized with DCs co-transduced with the IL-12 gene alone and mice immunized with DCs simultaneously co-transduced with the GM-CSF and IL-12 genes. Co-transduction of the GM-CSF and IL-12 genes may possibly elicit a more potent efficacy than that of the IL-12 gene alone when NK-resistant tumor cells are used.

In conclusion, vaccination therapy using genetically modified DCs expressing the TAA gene and Th 1-type cytokine genes resulted in the generation of efficient therapeutic immune responses against tumors, leading to a complete cure in s.c. tumor models. In particular, co-transduction of the IL-12 gene into DCs expressing TAA is crucial in this vaccination therapy in terms of the activation of DCs, NK cells, CD4 ${ }^{+}$ $\mathrm{T}$ cells and $\mathrm{CD}^{+} \mathrm{T}$ cells, which are fully involved in antitumor immunity. This strategy may be useful for clinical application as a cancer vaccine therapy in the future.

\section{Acknowledgements}

This study was supported by Grant-in-Aid no. 15591354 from the Ministry of Education, Culture, Sports, Science and Technology of Japan.

\section{References}

1. Austyn JM: New insights into the mobilization and phagocytic activity of dendritic cells. J Exp Med 183: 1287-1292, 1996.

2. Banchereau J and Steinman RM: Dendritic cells and the control of immunity. Nature 392: 245-252, 1998.

3. Gately MK, Wolitzky AG, Quinn PM and Chizzonite R: Regulation of human cytolytic lymphocyte responses by interleukin-12. Cell Immunol 143: 127-142, 1992.

4. Nakamura M, Iwahashi M, Nakamori M, Ueda K, Ojima T, Naka T, Ishida $\mathrm{K}$ and Yamaue $\mathrm{H}$ : Dendritic cells transduced with tumor-associated antigen gene elicit potent therapeutic antitumor immunity: comparison with immunodominant peptide-pulsed DCs. Oncology 68: 163-170, 2005.

5. Jonuleit H, Knop J and Enk AH: Cytokines and their effects on maturation, differentiation and migration of dendritic cells. Arch Dermatol Res 289: 1-8, 1996.

6. Sallusto F and Lanzavecchia A: Efficient presentation of soluble antigen by cultured human dendritic cells is maintained by granulocyte/macrophage colony-stimulating factor plus interleukin 4 and downregulated by tumor necrosis factor $\alpha$. J Exp Med 179: 1109-1118, 1994.

7. Nakamura M, Iwahashi M, Nakamori M, Ueda K, Matsuura I, Noguchi K and Yamaue H: Dendritic cells genetically engineered to simultaneously express endogenous tumor antigen and granulocyte macrophage colony-stimulating factor elicit potent therapeutic antitumor immunity. Clin Cancer Res 8: 2742-2749, 2002.

8. Braun SE, Chen K, Foster RG, Kim CH, Hromas R, Kaplan MH, Broxmeyer HE and Cornetta K: The CC Chemokine CK B-11/ MIP-3 $3 /$ ELC/Exodus 3 mediates tumor rejection of murine breast cancer cells through NK cells. J Immunol 164: 4025-4031, 2000. 
9. Kobayashi M, Fitz L, Ryan M, Hewick RM, Clark SC, Chan S, Loudon R, Sherman F, Perussia B and Trinchieri G: Identification and purification of natural killer cell stimulatory factor (NKSF); a cytokine with multiple biologic effects on human lymphocytes. J Exp Med 170: 827-845, 1989.

10. Stern AS, Podlaski FJ, Hulmes JD, Pan YC, Quinn PM, Wolitzky AG, Familletti PC, Stremlo DL, Truitt T, Chizzonite R and Gately MK: Purification to homogeneity and partial characterization of cytotoxic lymphocyte maturation factor from human B-lymphoblastoid cells. Proc Natl Acad Sci USA 87: 6808-6812, 1990.

11. Hochrein H, Shortman K, Vremec D, Scott B, Hertzog P and O'Keeffe M: Differential production of IL-12, IFN-alpha, and IFN-gamma by mouse dendritic cell subsets. J Immunol 166: 5448-5455, 2001.

12. Stober D, Schirmbeck R and Reimann J: IL-12/IL-18-dependent IFN-gamma release by murine dendritic cells. J Immunol 167: 957-965, 2001.

13. Cella M, Scheidegger D, Palmer-Lehmann K, Lane P, Lanzavecchia A and Alber G: Ligation of CD40 on dendritic cells triggers production of high levels of interleukin-12 and enhances T cell stimulatory capacity: T-T help via APC activation. J Exp Med 184: 747-752, 1996.

14. Pan PY, Gu P, Li Q, Xu D, Weber K and Chen SH: Regulation of dendritic cell function by NK cells: mechanisms underlying the synergism in the combination therapy of IL-12 and 4-1BB activation. J Immunol 172: 4779-4789, 2004.

15. Fukao T, Matsuda S and Koyasu S: Synergistic effects of IL-4 and IL-18 on IL-12-dependent IFN- $\gamma$ production by dendritic cells. J Immunol 164: 64-71, 2000.

16. Huang AY, Gulden PH, Woods AS, Thomas MC, Tong CD, Wang W, Engelhard VH, Pasternack G, Cotter R, Hunt D, Pardoll DM and Jaffee EM: The immunodominant major histocompatibility complex class I-restricted antigen of a murine colon tumor derives from an endogenous retroviral gene product. Proc Natl Acad Sci USA 93: 9730-9735, 1996.

17. Ueda K, Iwahashi M, Nakamori M, Nakamura M, Matsuura I, Yamaue $\mathrm{H}$ and Tanimura $\mathrm{H}$ : Carcinoembryonic antigen-specific suicide gene therapy of cytosine deaminase/5-fluorocytosine enhanced by the Cre/loxP system in the orthotopic gastric carcinoma model. Cancer Res 61: 6158-6162, 2001

18. Ueda K, Iwahashi M, Matsuura I, Nakamori M, Nakamura M, Ojima T, Naka T, Ishida K, Matsumoto K, Nakamura T and Yamaue $\mathrm{H}$ : Adenoviral-mediated gene transduction of the hepatocyte growth factor antagonist, NK4, suppresses peritoneal metastases of gastric cancer in nude mice. Eur J Cancer 40: 2135-2142, 2004.

19. Kay MA, Graham F, Leland F and Woo SL: Therapeutic serum concentrations of human alpha 1-antitrypsin after adenoviralmediated gene transfer into mouse hepatocytes. Hepatology 21: 815-819, 1995.

20. Lutz MB, Kukutsch N, Ogilvie AL, Rossner S, Koch F, Romani N and Schuler G: An advanced culture method for generating large quantities of highly pure dendritic cells from mouse bone marrow. J Immunol Methods 223: 77-92, 1999.

21. Iwahashi M, Tanimura H, Yamaue H, Tsunoda T, Tani M, Tamai M, Noguchi K and Hotta T: Defective autologous mixed lymphocyte reaction (AMLR) and killer activity generated in the AMLR in cancer patients. Int J Cancer 51: 67-71, 1992.

22. Rissoan MC, Soumelis V, Kadowaki N, Grouard G, Briere F, de Waal Malefyt R and Liu YJ: Reciprocal control of T helper cell and dendritic cell differentiation. Science 283: 1183-1186, 1999.
23. Albert ML, Jegathesan M and Darnell RB: Dendritic cell maturation is required for the cross-tolerization of $\mathrm{CD}^{+} \mathrm{T}$ cells. Nat Immunol 2: 1010-1017, 2001.

24. Reis e Sousa C, Hieny S, Scharton-Kersten T, Jankovic D, Charest $\mathrm{H}$, Germain $\mathrm{RN}$ and Sher A: In vivo microbial stimulation induces rapid CD40L-independent production of IL-12 by dendritic cells and their redistribution to T cell areas. J Exp Med 186: 1819-1829, 1997.

25. Hill HC, Conway TF Jr, Sabel MS, Jong YS, Mathiowitz E, Bankert RB and Egilmez NK: Cancer immunotherapy with interleukin 12 and granulocyte-macrophage colony-stimulating factor-encapsulated microspheres: coinduction of innate and adaptive antitumor immunity and cure of disseminated disease. Cancer Res 62: 7254-7263, 2002.

26. Wang J, Snider DP, Hewlett BR, Lukacs NW, Gauldie J, Liang $\mathrm{H}$ and Xing Z: Transgenic expression of granulocytemacrophage colony-stimulating factor induces the differentiation and activation of a novel dendritic cell population in the lung. Blood 95: 2337-2345, 2000.

27. Nishimura N, Nishioka Y, Shinohara T and Sone S: Enhanced efficiency by centrifugal manipulation of adenovirus-mediated interleukin 12 gene transduction into human monocyte-derived dendritic cells. Hum Gene Ther 12: 333-346, 2001.

28. Raulet DH: Interplay of natural killer cells and their receptors with the adaptive immune response. Nat Immunol 5: 996-1002, 2004.

29. Gerosa F, Baldani-Guerra B, Nisii C, Marchesini V, Carra G and Trinchieri G: Reciprocal activating interaction between natural killer cells and dendritic cells. J Exp Med 195: 327-333, 2002.

30. Fernandez NC, Lozier A, Flament C, Ricciardi-Castahndi P, Bellet D, Suter M, Perricaudet M, Tursz T and Maraskovsky E: Dendritic cells directly trigger NK cell functions: cross-talk relevant in innate anti-tumor immune responses in vivo. Nat Med 5: 405-411, 1999.

31. Zatloukal K, Schneeberger A, Berger M, Schmidt W, Koszik F, Kutil R, Cotton M, Wagner E, Buschle M and Maass G: Elicitation of a systemic and protective anti-melanoma immune response by an IL-2-based vaccine. Assessment of critical cellular and molecular parameters. J Immunol 154: 3406-3419, 1995.

32. Ossendorp F, Toes RE, Offringa R, van der Burg SH and Melief CJ: Importance of CD4 ${ }^{+} \mathrm{T}$ helper cell responses in tumor immunity. Immunol Lett 74: 75-79, 2000.

33. Heike M, Schlaak J, Schulze-Bergkamen H, Heyl S, Herr W, Schmitt U, Schneider PM and Meyer zum Buschenfelde KH: Specificities and functions of CD4 ${ }^{+}$HLA class II-restricted T cell clones against a human sarcoma: evidence for several recognized antigens. J Immunol 156: 2205-2213, 1996

34. Tatsumi T, Huang J, Gooding WE, Gambotto A, Robbins PD, Vujanovic NL, Alber SM, Watkins SC, Okada H and Storkus WJ: Intratumoral delivery of dendritic cells engineered to secrete both Interleukin (IL)-12 and IL-18 effectively treats local and distant disease in association with broadly reactive Tc 1-type immunity. Cancer Res 63: 6378-6386, 2003.

35. Manetti R, Parronchi P, Giudizi MG, Piccinni MP, Maggi E, Trinchieri $G$ and Romagnani S: Natural killer cell stimulatory factor [interleukin 12 (IL-12)] induces T helper type 1 (Th 1)specific immune responses and inhibits the development of IL-4producing Th cells. J Exp Med 177: 1199-1204, 1993.

36. Van den Broeke LT, Daschbach E, Thomas EK, Andringa G and Berzofsky JA: Dendritic cell-induced activation of adaptive and innate antitumor immunity. J Immunol 171: 5842-5452, 2003. 